\title{
LE RYTHME NYCTHÉMÉRAL DE LA CONCENTRATION EN MINÉRAUX DE L'URINE ET DES FÈCES CHEZ LES BOVINS
}

PAR

\section{P. LARVOR et M. BROCHART (1)}

Laboratoire de Zootechnie, École nationale Vétérinaire, Alfort

\section{I. - INTRODUCTION}

I'existence d'un rythme nycthéméral de la concentration urinaire en diverses substances et de la sécrétion d'urine est établie depuis longtemps. Parmi les auteurs qui ont étudié de tels rythmes excrétoires, on peut citer Ifeathes (IgO6-7), Simpson (I924-26-29), NORN (I929), MANCheSTER (I933), Welsh (I938) dont les recherches ont porté sur l'excrétion de l'acide urique, du chlore, du sodium et du potassium.

Ces premiers travaux ont permis d'établir que l'urine est, d'une façon générale, plus concentrée la nuit, mais que la diminution de la sécrétion urinaire compense, et au-delà, l'augmentation de concentration, le résultat définitif étant une baisse de l'excrétion globale des matières solides et de l'eau.

I'étude du rythme nycthéméral chez les bovins est intéressante au double point de vue pratique et théorique.

I $^{\circ} \mathrm{Au}$ point de vue pratique, parce qu'elle va permettre d'examiner le caractère représentatif d'un échantillon unique d'urine ou de fécès par rapport à l'élimination urinaire ou fécale de vingt-quatre heures d'un animal.

Lors de l'exploration clinique du métabolisme minéral, dans les conditions pratiques, il n'est guère possible d'effectuer qu'un seul prélèvement d'urine ou éventuellement de fèces; il est donc essentiel de savoir le degré de confiance que l'on peut accorder à une telle exploration. Par ailleurs, la connaissance du rythme nycthéméral de l'élimination de divers éléments minéraux pourrait faciliter la conduite de recherches à long terme sur la nutrition minérale. En effet, de nombreuses difficultés techniques rendent la réalisation d'un bilan complet très difficile chez les bovins, surtout sur une période supérieure à quelques semaines. La mise d'une vache dans une stalle à métabolisme est très " stressante " pour

(1) Avec la collaboration technique de Mme N. Berthelot et M. C. Roth. 
l'animal, et on ne peut avoir la certitude que les renseignements recueillis dans ces conditions, c'est-à-dire chez des animaux en état d'hyperfonctionnement surrénalien chronique, correspondent à peu de chose près à la physiologie normale des bovins. Il existe bien des dispositifs moins "stressants" que les cages à métabolisme, mais ceux-ci nécessitent un équipement très important et un personnel nombreux et qualifié, permettant une surveillance constante.

Une expérimentation de longue durée (plusieurs mois) ne peut donc guère être faite que sur des échantillons prélevés périodiquement, à la même heure, et il est très utile de pouvoir déterminer la confiance qu'il est possible d'accorder aux résultats obtenus de cette façon, et de savoir s'il existe des heures privilégiées de prélèvement, où l'écart par rapport à la moyenne est plus faible que pour d'autres heures. Il est évident que la validité des chiffres ainsi obtenus dépend essentiellement de la régularité des rythmes nycthéméraux pour la substance considérée.

$2^{\circ} \mathrm{Au}$ point de vue théorique, parce que le rythme nycthéméral urinaire n'a guère fait l'objet de recherches chez les bovins, et, qu'à notre connaissance, l'élimination fécale n'a été envisagée sous cet angle dans aucune espèce.

\section{LE PROBLEME DU DETERMINISME DU RYTHME NYCTHEMERAL}

Cette question a fait l'objet de nombreux travaux récents. Pour AZERAD et Coll. (I953), les variations nycthémérales sont beaucoup plus le fait de phénomènes tubulaires rénaux que celui de variations de la filtration glomérulaire ; en effet, la clearance de la créatinine endogène (essentiellement fonction des variations de la filtration glomérulaire, car cette substance n'est ni sécrétée, ni réabsorbée au niveau du tube urinifère), bien que soumise à des variations importantes au cours de 24 heures, ne présente aucune rythmicité.

D'autres auteurs (BORST et de VRIES, I950 ; SiRota et Coll., I950 ; Ghata, I954; Metz et Mours-Laroche, 1954; Noble, 1957) ont précisé les modalités du rythme nycthéméral, mais par des études sur l'homme, chez lequel l'alternance sommeil-veille est très marquée. Les phénomènes ne sont pas comparables chez des animaux comme le chien, qui présente plusieurs périodes de sommeil et de veille dans une même journée. Ceci est à rapprocher des résultats de Kayser (I949), qui a montré que le rythme nycthéméral n'existe pas chez. le nouveau-né et qu'il met un certain temps à s'établir. Ghata et ReINBERG (I954) ont établi que le rythme nycthéméral est largement influencé par les conditions géographiques et saisonnières. I'interprétation globale du déterminisme de ce rythme a fait l'objet d'hypothèses nombreuses et souvent 
divergentes. Pour StTanbury et Thomson (I95I), le rythme excrétoire pourrait être dû à des changements acidobasiques cycliques, liés en particulier à l'hyperventilation du réveil, mais ce point de vue reste purement hypothétique, et la tendance actuelle va plutôt dans le sens d'une interprétation endocrinologique.

Un fait ne peut plus guère être contesté : l'activité rythmique de l'hypothalamus gouverne l'ensemble de ces phénomènes. Dès I933, JOREs lui attribue les variations nycthémérales du volume urinaire, faisant valoir qu'elles présentent un parallélisme frappant avec le rythme du sommeil ou de la température. MiLLs (I95I) expose un point de vue analogue, et ces deux auteurs font remarquer que, si le rythme hypothalamique est, en dernière analyse, sous la dépendance des facteurs externes, il possède néanmoins une relative autonomie et peut persister une huitaine de jours, alors même que le rythme de vie active a été inversé, avant de s'inverser lui-même. Pour Rosenbaum et Coll. (1952), les troubles du rythme nycthéméral induits par la cortisone (disparition ou inversion du rythme) sont à rapprocher des troubles cérébraux prononcés (en particulier troubles du sommeil) qu'entraîne cette hormone. Enfin, les notions récentes sur la physiologie du complexe hypothalamo-posthypophysaire, ainsi que l'action inhibitrice de l'hydrocortisone sur l'hypothalamus (voir la revue de Stutinski, I957), ne laissent subsister aucun doute quant au rôle prépondérant de cette région dans le déterminisme du cycle quotidien de l'activité de l'organisme dans tous les domaines.

Il reste à déterminer par quels intermédiaires cette action se fait sentir sur le rein. Pour les uns, l'activité de la posthypophyse commande pour l'essentiel ces variations. D'ARCY HAR'T et VERNEY (1934) ayant mis en évidence l'inhibition de la sécrétion posthypophysaire dans la diurèse provoquée ou spontanée, il semblait logique de lui attribuer les variations nycthémérales du flux urinaire. KLEITMAN (I939-I949), Mills (I95I), Rosenbaum et Coll. (I952) lui concèdent un rôle quasi exclusif. Cependant, Goldman et Luchsinger (1956) ne trouvent pas une relation directe entre la concentration urinaire et sa teneur en A. D. H; ils émettent 1'hypothèse que cette dernière hormone pourrait ne régler que le niveau général de la diurèse, les variations nycthémérales étant sous l'influence d'un autre mécanisme, et la réaction à la surcharge hydrique étant un mécanisme d'urgence. Cependant, ils reconnaissent euxmêmes la faiblesse de l'hypothèse, vu la rareté du principe antidiurétique urinaire, les difficultés encore rencontrées dans le dosage, et la possibilité non exclue d'une réabsorption de 1'A. D. H. par la muqueuse vésicale.

Pour d'autres auteurs cependant, le rôle de la surrénale dans le cycle quotidien d'élimination est loin d'être négligeable. La notion d'un rythme corticosurrénal est solidement établie sur deux séries de preuves:

$I^{\circ}$ Preuves indirectes, par numération des éosinophiles ou des lympho- 
cytes sanguins (test de ThORNE), dont la diminution coïncide avec la mise en circulation des corticoïdes, à tel point que la mesure de cette chute a pu être utilisée comme technique de dosage (Coste, IAURENT et Delbarre, I95I ; Bibile, I953). Ainsi Elmadjian et Pincus (I946) établissent que les éosinophiles sont à un taux minimum entre 6 et Io heures puis remontent progressivement jusqu'au lendemain (il y a une régression linéaire temps-éosinophiles); de même Rud (I947), Herbert et DE Vries (I949), Halberg et Coll. (I95 I-53), Doe, Flink et Goodselit, (I956) aboutissent à des conclusions analogues, (tant pour les lymphocytes que pour les éosinophiles).

$2^{\circ}$ Preuves directes, par mesure de l'excrétion des corticoïdes au cours de 24 heures. Bachman et Coli. (I94I), chez la femme, ne parvinrent pas à mettre en évidence une différence d'excrétion des corticoides entre 8 heures et 20 heures; cependant, on peut objecter que les prélèvements urinaires devaient, dans les deux cas, recouvrir des périodes d'activité analogues. Par contre, Pincus (I943) observe un rythme excrétoire très net des $I 7$ cétostéroïdes chez l'homme, l'excrétion étant maximum le matin et minimum la nuit. Ces travaux ont été maintes fois confirmés depuis (Pincus et Coll., I948 ; LAIDLAW et Coll., I954; Tyler et Coll, I954 ; ENGEl et Coll., r955 ; Forsham et Coll., I955; DoE et Coll., I956; VestergaARD et LEVERETT, I957).

On connaît par ailleurs les altérations profondes du rythme nycthéméral provoquées par la maladie d'Addison (Rosenbaum, I952; AzERAD et Coll., I957), ou par la surrénalectomie (SImpson et TAIT, I950) qui entraîne la suppression du rythme chez le rat à un régime normal ; cependant, l'administration de quantités importantes de sel est susceptible de rétablir le rythme nycthéméral. Dans ces conditions, le rapport des quantités de sodium excrétées par unité de temps le jour et la nuit est le même, que les animaux soient surrénalectomisés ou non, la seule différence étant dans les valeurs absolues de sodium excrété. Il semblerait donc que le cortex jouerait un rôle limité au maintien d'un équilibre minéral correct, nécessaire au jeu normal des fluctuations de 1'A. D. H Pour beaucoup d'auteurs, toutefois, les corticoides jouent un rôle direct dans le contrôle du flux urinaire, et on peut rappeler à ce sujet la notion d'antagonisme surrénalo-posthypophysaire, défendue par SILVETTE et BRITTON (1933-38) et CoREy et Coll. (1939) et assez généralement admise actuellement, l'antagonisme sur la diurèse étant susceptible d'interprétations diverses- :

Io L'hypofonctionnement surrénalien entraînerait l'augmentation du taux d'A. D. H. circulant, ainsi que l'affirment Birnie et Coll. (I949) ; mais cette notion a été vivement critiquée par AMEs et VAN Dyke $\left(\mathrm{I} 95^{\left.\mathrm{I}-5^{2}\right)}\right.$ et ne semble guère admise actuellement.

$2^{\circ}$ Pour Birnie et Coll. (I950), il y aurait une action de la cortisone 
sur la sensibilité à l'A. D. H. de l'effecteur rénal. GuINnEB.tult et Morel (I957) ont montré que la surrénalectomie chez le rat se traduit au niveau rénal par la diminution $\mathrm{du}$ gradient de concentration corticopapillaire des électrolytes, qui reste de plus sensiblement constant, et ne réagit plus notamment à la surcharge en eau ou à la surcharge en sel. Le rôle des corticoïdes sur le pouvoir de concentration et de dilution de l'urine par le rein pourrait s'expliquer par leur action sur l'établissement d'un gradient de pression osmotique entre le cortex rénal et la papille, selon un mécanisme encore à déterminer.

$3^{\circ}$ Les glucocorticoïdes catalysent la destruction hépatique de 1'A. D. H., ainsi que l'on montré in vitro les travaux de HELLER et URBAN (I935), d'après qui, bien que le rein et le sang inactivent aussi 1'A. D. H., le tissu hépatique a une action plus puissante. RALLI et Coll. (I945) émettent l'hypothèse selon laquelle, si l'on trouve beaucoup d'A. D. H. dans les urines de malades à dysfonctionnement hépatique c'est parce que cette hormone n'est plus détruite par le foie dans ces conditions. Eversole et Coll. (I949), Dicker et Greenbatm (I954-55-56), Ginsburg (I954), Hechter et Coll. (I956), Helier et ZaIdi (I957) confirment ces notions tant in vivo que in vitro. Il est certain que l'analogie profonde qui existe entre la courbe de sécrétion des corticoïdes et celle du flux urinaire rend cette hypothèse très séduisante; d'ailleurs, pour STUTINSKy (I957), les corticoïdes pourraient influencer fortement la diurèse par la destruction de l'A. D. H., sans nécessairement modifier le niveau plasmatique de cette hormone d'une façon manifeste; hypothèse qui prend en considération les travaux d'Ames et VAN DYKE, cités précédemment.

Nous envisagerons, lors de la discussion des résultats, quelques critiques à ces théories.

\section{III. - MATERIEL ET METHODES}

Nos recherches ont porté sur deux couples de jumelles vraisemblablement univitellines, de race normande. Le premier couple sera désigné par les chiffres I et III, le second, par les lettres E, et S. Six expériences ont été réalisées entre octobre 1957 et mars 1958 , à des intervalles de I mois environ. Chacune d'elles a porté sur deux animaux appartenant à des couples différents.

Les animaux ont été entretenus en stabulation permanente; ils recevaient directement la lumière par des fenêtres situées de face. I’abreuvement était automatique ; les repas étaient distribués à $9 \mathrm{~h}$ et $\mathrm{I} 7 \mathrm{~h}$, les rations du matin et du soir étant identiques.

Les prélèvements étaient faits de 4 en 4 heures (Io, I4, I8, 22, 2, 6 et ro heures, le lendemain). I,es fèces étaient prélevés dans le rectum, 
et l'urine directement, dans la vessie, à l'aide d'une sonde. Les fèces récoltés étaient desséchés à $1^{\prime}$ étuve à I0 $5^{\circ}$, après pesée, pour déterminer le taux de matière sèche. Les bouses sèches étaient broyées, et Ioo mg de broyat minéralisés au four à $550^{\circ}$ pendant $\mathrm{I} 6$ heures. I,e sodium et le potassium ont été dosés par spectrophotomètrie de flamme. Le chlore urinaire a été dosé par la méthode CHARPENTIER-VoLHAR'T, décrite par LOISELEUR. Le phosphore et le calcium ont été dosés selon les méthodes déjà décrites par l'un de nous (BrocharT, I957). I,es données obtenues ont été comparées avec les conditions météorologiques principales (température, pression atmosphérique, hygrométrie), qui nous ont été fournies par l'Observatoire Géophysique du Parc Saint-Maur, dont le microclimat se rapproche beaucoup du nôtre.

\section{IV. - RESULTATS}

\section{A. Existence d'un rythme nycthéméral.}

IO RYTHME LRINAIRE; :

I a figure I montre l'ensemble des résultats moyens pour l'urine. Ces courbes représentent, pour chaque heure de prélèvement, la moyenne des valeurs individuelles observées au cours des I2 expériences. Chaque valeur individuelle a été exprimée en pourcent par rapport à la moyenne des 7 valeurs du nycthémère correspondant, ceci a fin de donner la mêne importance relative aux différents nycthémères, les valeurs absolues pouvant varier de façon considérable d'un nycthémère à l'autre. Nous avons chaque fois représenté en pointillé le cœfficient de variation à chaque heure de prélèvement.

Il convient de rappeler que nos résultats sont qualitatifs (composition de l'urine aux différentes heures), mais non quantitatifs.

L'examen des courbes permet de dégager immédiatement la notion d'un rythme nycthéméral urinaire. La matière sèche de l'urine présente un minimum à Io heures et un maximum à 22 heures, avec une courbe assez régulière. I e fait que l'on n'observe pas tout à fait le retour au niveau initial peut s'expliquer par une légère décharge d'A. D. H. sous l'influence des cathétérismes répétés.

Le potassium présente le rythme de concentration le plus régulier, rythme d'ailleurs voisin de celui de la matière sèche urinaire. Cette régularité remarquable dans l'excrétion du potassium a déjà été signalée chez 1'homme par AzERAD et Coll., (I953).

Le calcium présente aussi un rythme assez régulier, suivant de près celui de la matière sèche, à l'exception d'une légère dépression vers 22 heures.

Le phosphore a un niveau moyen assez constant, dont les seules 
variations sont une diminution régulière et lente entre I4 et 6 heures, suivie d'une remontée rapide qui se place entre 6 et ro heures.

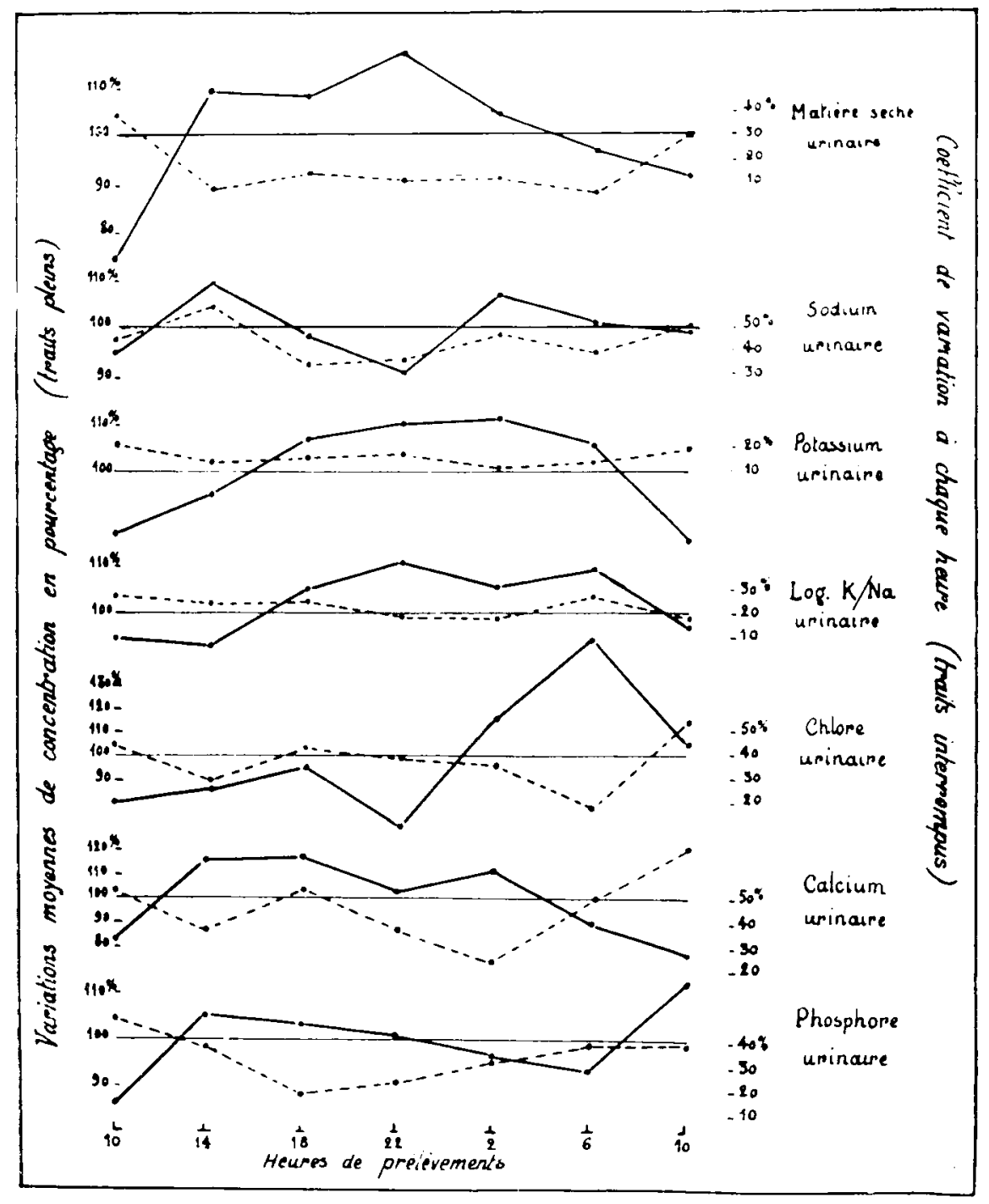

FIG. 1. - Variations uscthémérales de la concentration urinaire, et de son coefficient de variation.

Le sodium et le chlore présentent tous deux une courbe biphasique très nette, dont le minimum se situe vers 22 heures, les deux maxima étant légèrement décalés, cenx du sodium se produisant avant ceux du chlore.

La répartition des valeurs du rapport $\mathrm{K} / \mathrm{Na}$ ne suivant pas une 
courbe de Gauss, il a été nécessaire d'en donner une représentation logarithmique qui ramène ces valeurs à une répartition normale. Ce fait est d'ailleurs à rapprocher des travaux de CHERnik et Coll. (I950) qui ont

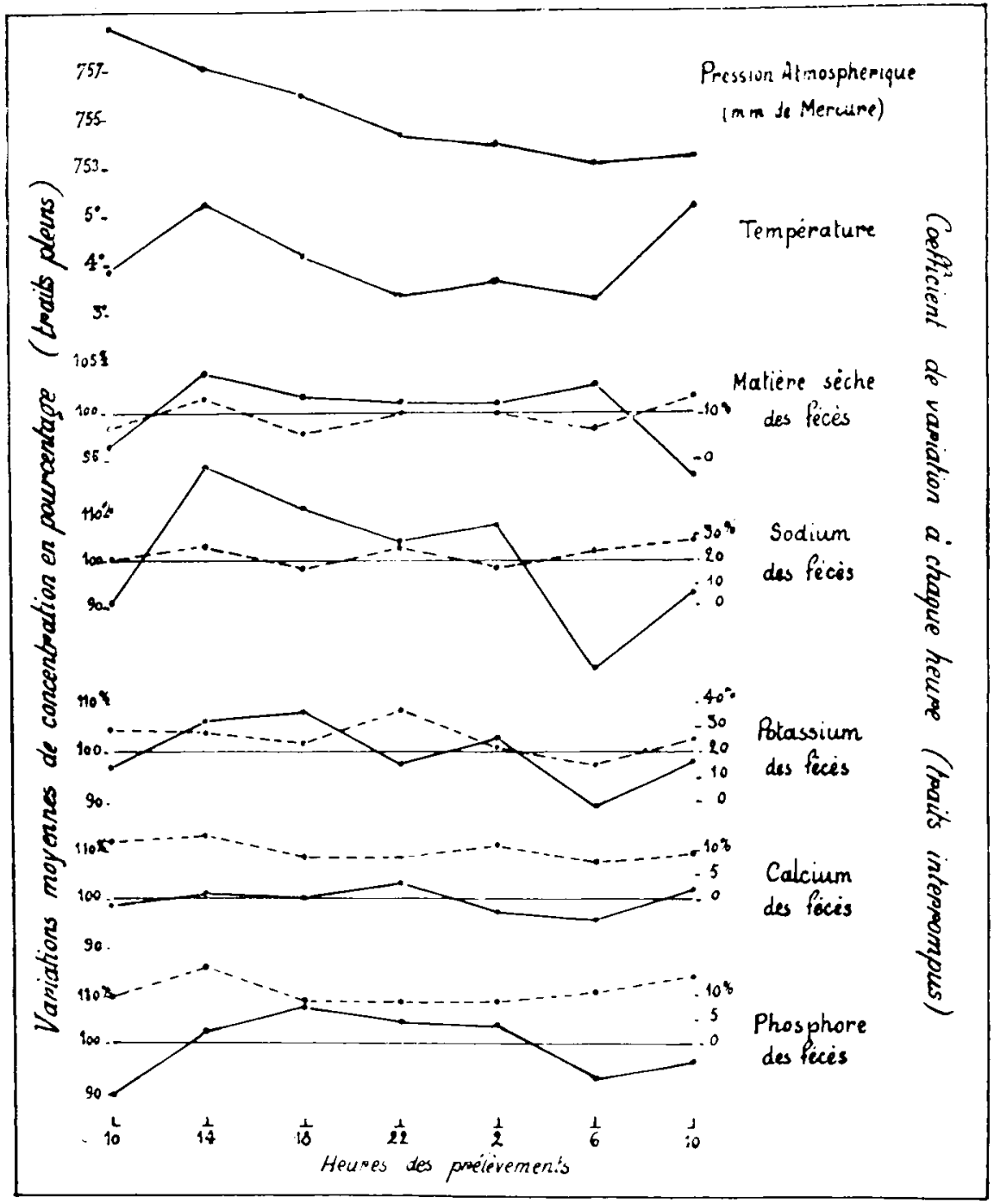

FIG. 2. -- Variations nycthémérales des conditions météorologiques, de la concentration fécale et de son coefficient de variation.

mis en évidence une relation linéaire hautement significative entre le logarithme de $\mathrm{K} / \mathrm{Na}$ et l'aldostérone urinaire chez l'homme. Dans ces conditions, il était permis d'espérer que la courbe du $\mathrm{I}_{4} \mathrm{og} \mathrm{K} / \mathrm{Na}$ urinaire nous donnerait une idée des variations nycthémérales de la sécrétion d'aldostérone. Nous discuterons plus loin de cette possibilité. Quoiqu'il 
en soit, la courbe obtenue présente un minimum diurne entre ro et $\mathrm{I}_{4}$ heures et un maximum nocturne entre 18 et 6 heures.

$2^{\circ}$ RYTHME FECAL :

La figure 2 montre les variations de la concentration fécale, ainsi que la courbe moyenne des températures et des pressions atmosphériques. Il en ressort très nettement la notion d'un rythme nycthéméral de la matière sèche fécale, dont la courbe est d'une régularité manifeste. La principale caractéristique en est une dilution nette des fèces vers Io heures, la concentration se maintenant en plateau entre I4 et 6 heures, avec une tendance à une très légère dépression nocturne. Cette dilution matinale des fèces est donc synchrone du flux maximum urinaire et de sa dilution maximum; son interprétation semble donc pouvoir faire appel à des mécanismes de même nature, que nous discuterons ultérieurement.

La teneur en minéraux des fèces est calculée en fonction des fèces deshydratés, ce qui explique qu'il n'y ait pas de rapports entre la courbe de matière sèche et celle des minéraux. La teneur en calcium se caractérise par sa régularité remarquable, les variations qui l'affectent semblant bien être fortuites. Les courbes du sodium, du potassium et du phosphore présentent une analogie frappante entre elles et avec la courbe moyenne des températures pendant les nycthémères. Il semble donc que l'élévation de la température diminue la résorption de $\mathrm{Na}, \mathrm{K}$ et $\mathrm{P}$, à moins qu'elle n'accroisse l'excrétion endogène de ces substances.

\section{B) Coefficient de variations aux différentes heures}

Le coefficient de variation, qui n'est autre qu'une estimation de la dispersion des valeurs autour de leur moyenne, va nous permettre de voir s'il existe tune heure privilégiée à laquelle il est préférable de faire les prélèvements pour qu'ils soient aussi représentatifs que possible.

En ce qui concerne les urines, on voit (fig. I) qu'il convient de ne pas prélever d'échantillons vers io heures (dispersion considérable de tous les facteurs étudiés). Les heures les plus favorables, tant du point de vue de la dispersion des valeurs que de la commodité de prélèvement, seront donc I4 heures (matière sèche, $\mathrm{Ca}, \mathrm{Cl}, \mathrm{K}$ ) et I8 heures ( $\mathrm{Na}$ etP). Dans la pratique, nous estimons que, pour obtenir des résultats représentatifs, il convient d'opérer à l'une de ces deux heures selon le but recherché. Il est à noter que les périodes où l'excrétion est stable semblent correspondre aux périodes oì l'activité endocrinienne est également stable; par contre, le début de la matinée, qui, selon les idées actuelles $(62,67)$, est une phase où l'activité endocrinienne évolue considérablement dans un temps assez court, fournit des chiffres extrêmement variables. En ce qui nous concerne, nos animaux recevant un repas à I7 heures, 
nous pensons qu'un prélèvement urinaire à $r 6$ heures est indiqué, ce qui permet d'escompter un coefficient de variation de $\mathrm{I} 2 \mathrm{p}$. Ioo pour la matière sèche, I $5 \mathrm{p}$. Ioo pour le potassium, 25 à $30 \mathrm{p}$. Ioo pour le phosphore, les coefficients de variation du chlore, du sodium et du calcium étant plus élevés, et difficilement estimables du fait de la répartition anormale des valeurs. I e coefficient de variation $d u \log \mathrm{K} / \mathrm{Na}$ reste assez constant et de l'ordre de 25 p. 100.

Cependant, le coefficient de variation, s'il permet de comparer le caractère représentatif des prélèvements effectués à des heures différentes, n'est pas suffisant pour estimer la signification d'une mesure isolée. En effet, il est manifeste qu'à égalité de variation, une mesure sera d'autant plus significative que les valeurs habituellement trouvées seront. plus dispersées. Dans le but d'estimer le degré de correspondance entre les valeurs isolées et les valeurs moyennes, nous avons calculé le coefficient de corrélation entre celles-ci et la mesure effectuée à l'heure où la variation est la plus faible.

La corrélation la plus remarquable est celle du $\mathrm{L}, \mathrm{og} \mathrm{K} / \mathrm{Na}$ urinaire $(\mathrm{r}=0,984)$. Nous trouvons ensuite la matière sèche urinaire $(\mathrm{r}=0,947)$, le phosphore $(r=0,883)$, le calcium $(\mathrm{r}=0,882)$, le sodium $(\mathrm{r}=0,874)$, le chlore $(r=0,829)$ et le potassium $(r=0,754)$. Les limites de signification sont, pour Io degrés de liberté, $r=0,708$ (à $\mathrm{I}$ p. Ioo) et $r=0,576$ (à 5 p. roo). Il en résulte que tous ces coefficients sont évidemment significatifs, mais que les chiffres fournis par une mesure isolée de chlore ou de potassium urinaire ne sont guère exploitables pratiquement. Le cas du potassium illustre bien le fait que le coefficient de variation, (assez faible pour $\mathrm{K}$, I 5 p. Ioo) ne suffit pas à évaluer la signification d'un dosage, si, par ailleurs, les limites extrêmes des fluctuations d'un jour à l'autre sont assez resserrées. En effet, l'erreur sur la concentration urinaire en potassium, pour une heure déterminée, sera assez faible par rapport à la concentration moyenne du nycthémère correspondant, mais très forte relativement aux variations de la kaliurie d'un jour à l'autre ou d'un individu à l'autre.

En tenant compte des éléments précédents, il est possible de déterminer si deux mesures de la teneur en minéraux de l'urine ou les fèces, prélevés le même jour sur deux animaux différents ou à des jours différents sur le même animal, sont significativement différentes.

La dispersion des valeurs est beaucoup plus constante pour les fèces au cours du nycthémère, et l'on peut voir que l'heure du prélèvement n'a guère d'importance, sauf si l'on veut doser le sodium et le potassium, auquel cas il est préférable d'opérer vers i 8 heures.

Dans ces conditions, les cœfficients de variation seront : matière sèche 5 à Io p. IOo, calcium Io p. IOO, phosphore Io à I 5 p. IOo, sodium 20 p. IOO, potassium 25 p. I00. 
Les coefficients de corrélation entre la mesure à 18 heures et la moyenne du nycthémère sont : $\mathrm{r}=0,950$ pour le potassium, $\mathrm{r}=0,88 \mathrm{I}$ pour le calcium, $\mathrm{r}=0,878$ pour le phosphore, $\mathrm{r}=0,844$ pour le sodiun et $r=0,699$ pour la matière sèche.

\section{C) Corrélations entre nycthémères différents.}

Il nous a paru intéressant d'envisager les corrélations entre les moyennes nycthémérales des douze expériences, les chiffres moyens obtenus étant certainement représentatifs de la concentration moyenne réelle au cours du nycthémère. Le tableau I donne une récapitulation de ces moyennes.

\section{$I^{\circ}$ RAPPORTS ENTRE Ca ET P URINAIRES.}

L'examen du tableau I permet de dégager immédiatement la notion d'une variation inverse du calcium et du phosphore urinaires. Cependant, la régression en cause n'est certainement pas linéaire, mais il semble que le produit $\mathrm{Ca} \times \mathrm{P}$ tende à être constant. La relation serait donc du type $\mathrm{Y}=\frac{n}{\mathrm{X}}$ (fig 3 ). Un examen plus attentif des données nous montre une relation nette entre la matière sèche urinaire et le produit $\mathrm{Ca} \times \mathrm{P}$ (fig 3.). I a Corrélation est : $\mathrm{r}=-0,8 \mathrm{I} 9$, significative à $\mathrm{I} p$. Ioo, et la régression linéaire : $\mathrm{Ca} \times \mathrm{P}=0$,o00 I52 M. S. + o,or 54 (M. S. représente la matière sèche urinaire pour I 000 ).

Il en résulte que l'on peut calculer un produit $\mathrm{Ca} \times \mathrm{P}$ corrigé évalué pour une matière sèche moyenne, soit $65 \mathrm{p}$. I ooo. Ce produit $\mathrm{Ca} \times \mathrm{P}$ corrigé est évidemment beaucoup plus constant que le produit brut. I a figure 4 montre les variations de $\mathrm{Ca} \times \mathrm{P}$ brut en fonction de $\mathrm{Ca}$ et de $\mathrm{P}$. Il en résulte que le produit $\mathrm{Ca} \times \mathrm{P}$ est relativement indépendant de $\mathrm{Ca}$, mais varie dans le même sens que, c'est-à-đire qui la correction du produit $\mathrm{Ca} \times \mathrm{P}$, due aux variations de la teneur en matière sèche de l'urine, doit porter sur $\mathrm{P}$ et non sur $\mathrm{Ca}$. Cette correction peut être exprimée par la formule :

$$
\mathrm{P} \text { corrigé }=\mathrm{P}+\frac{(\text { Matière Sèche }-65) 0,000 \mathrm{I} 5^{2}}{\mathrm{Ca}}
$$

Cette correction permet de dresser la courbe de corrélation $\mathrm{Ca}-\mathrm{P}$ (figure 5), qui, comme on le voit, se rapproche beaucoup de l'hyperbole théorique $\mathrm{Ca}=\frac{0,0055}{\mathrm{P}}$ Cependant, il semble qu'on n'ait pas véritablement affaire à une hyperbole, mais à deux types différents de courbes qui se coupent. Le point critique paraît être situé aux environs des valeurs de $\mathrm{Ca}$ et $\mathrm{P}$ urinaires de respectivement $0,03 \mathrm{p}$. I ooo et $0,09 \mathrm{p}$. rooo. La liaison de $\mathrm{Ca}$ et $\mathrm{P}$ semble totalement modifiée de part et d'autre de ce point, ce qui cadrerait très bien avec l'hypothèse d'un seuil de sécrétion 


\begin{tabular}{|c|c|c|c|c|c|c|c|c|c|}
\hline & \multirow{2}{*}{ 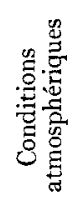 } & $\stackrel{\circ}{\epsilon}$ & $\vec{E}$ & gु & $\stackrel{2}{2}$ &  & $\stackrel{y}{z}$ & $\hat{\imath}$ & \\
\hline & & $\begin{array}{l}\dot{4} \\
\dot{4}\end{array}$ & $\underset{\hat{0}}{\hat{b}}$ & $\begin{array}{l}\text { a } \\
\text { in } \\
\end{array}$ & 苛 & 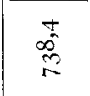 & 竎 & 출 & \\
\hline \multirow{17}{*}{ 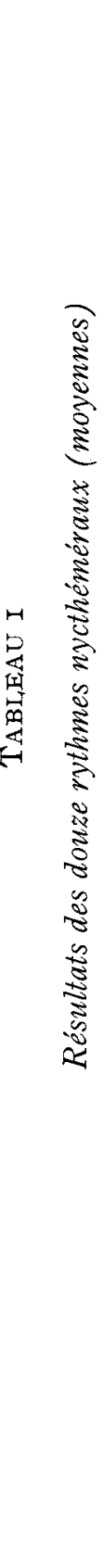 } & \multirow{5}{*}{ 怤 } & $a$ & $\mid \begin{array}{ll}\infty & 2 \\
\hat{H} & 2\end{array}$ & $\frac{2}{3}=$ & $\mid \begin{array}{ll}\infty & 0 \\
& 0\end{array}$ & 占 & 寽売 & 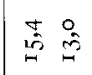 & \\
\hline & & $\pi$ & 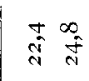 & 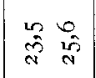 & $4=$ & 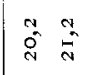 & 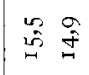 & 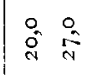 & \\
\hline & & $\because$ & $\mid \begin{array}{ll}\infty & 0 \\
0 & 0 \\
i n & 0\end{array}$ & \begin{tabular}{ccc}
0 & 0 \\
0 & 0 \\
\hdashline & 0 \\
\hdashline
\end{tabular} & $\begin{array}{c}q \\
q \\
\sigma^{2}\end{array}$ & 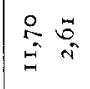 & 员哭 & $\begin{array}{ll}\overrightarrow{2} & = \\
a^{2} & =\end{array}$ & \\
\hline & & 孚 & $\mid \begin{array}{ll}\infty & 0 \\
0 & 5 \\
0 & 5\end{array}$ & $\mid \begin{array}{ll}\infty & 0 \\
\infty & 0 \\
c i & \alpha\end{array}$ & $\Xi$ & 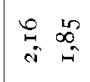 & 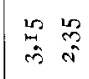 & 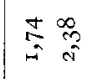 & \\
\hline & & $\frac{n}{2}$ & $\because 8$ & 芯菑 & $\stackrel{8}{2}$ & 옴 $\stackrel{\infty}{5}$ & $\stackrel{\circ}{\infty}$ & $\stackrel{0}{0}$ & \\
\hline & \multirow{10}{*}{ 总 } & E & $\mid \begin{array}{ll}\infty & 0 \\
0 & 0 \\
0 & 0\end{array}$ & $\stackrel{8}{:}$ & 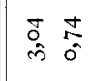 & 象 & 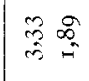 & 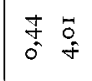 & \\
\hline & & م. & 11 & 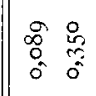 & $\begin{array}{lll}0 & 0 \\
0 & 0 \\
0 & 0 \\
0 & 0\end{array}$ & 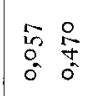 & 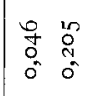 & 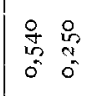 & \\
\hline & &  & 11 & 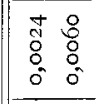 & $\begin{array}{lll}0 & 0 \\
0 & 0 \\
0 & 0 \\
0 & 0 \\
0 & 0\end{array}$ & $\begin{array}{ll} & 0 \\
0 & 0 \\
0 & 0 \\
0 & 0 \\
0 & 0 \\
\end{array}$ & $\begin{array}{ll} & 9 \\
0 & 0 \\
0 & 0 \\
0 & 0 \\
0 & 0\end{array}$ & $\begin{array}{lll}0 & 1 \\
0 & 0 \\
0 & 0 \\
0 & 0 & 0 \\
\end{array}$ & \\
\hline & & 2 & $\mid \begin{array}{ll}7 & 0 \\
0 & 0 \\
0 & 0 \\
0 & 0 \\
0 & 0\end{array}$ & $\mid \begin{array}{ll}c & 0 \\
0 & 0 \\
0 & 0 \\
0 & 0\end{array}$ & $\mid \begin{array}{ll}\widetilde{0} & 0 \\
0 & + \\
0 & 0 \\
0 & 0\end{array}$ & $\begin{array}{cc}\infty & 8 \\
0 & 0 \\
0 & 0 \\
0 & 0\end{array}$ & $\begin{array}{ll}\overrightarrow{1} & 8 \\
0 & 0 \\
0 & 0\end{array}$ & $\mid \begin{array}{cc}0 & 2 \\
0 & 2 \\
0 & 2 \\
0 & 2\end{array}$ & \\
\hline & & $\tilde{ت}$ & $\mid \begin{array}{lll}0 & 3 \\
0 & 0 \\
0 & 0 & 0\end{array}$ & 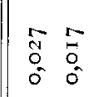 & 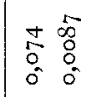 & $\begin{array}{cc} \pm & 0 \\
0 & 0 \\
0 & 0 \\
0 & 0\end{array}$ & 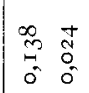 & $\mid \begin{array}{lll}\hat{1} & \overrightarrow{1} \\
0 & 0 \\
0 & 0 & 0 \\
0\end{array}$ &  \\
\hline & & $\begin{array}{l} \\
x \\
x \\
0 \\
\end{array}$ & $\mid \begin{array}{lll} & 0 \\
0 & 0 \\
0 & 0 \\
0 & 0 \\
0 & 0 & 0 \\
0\end{array}$ & $\mid \begin{array}{ll}0 & 0 \\
0 & 0 \\
0 & 0 \\
0 & 0 \\
0 & 0\end{array}$ & $\begin{array}{ll} & 0 \\
5 & 0 \\
0 & 0 \\
0 & 0 \\
0 & 0\end{array}$ & $\begin{array}{|ll|}0 & 0 \\
0 & 0 \\
0 & 0 \\
0 & 0 \\
0 & 0 \\
\end{array}$ & $\begin{array}{ll}3 & 0 \\
0 & 0 \\
0 & 0 \\
0 & 0 \\
0 & 0\end{array}$ & $\mid \begin{array}{ll} & \\
0 & 0 \\
0 & 0 \\
0 & 0 \\
0 & 0 \\
0\end{array}$ & : \\
\hline & & $90 \frac{50}{9}$ & 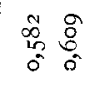 & 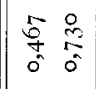 & 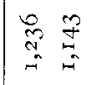 & 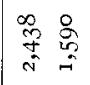 & 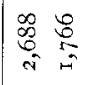 & $\mid$\begin{tabular}{ll}
0 & 0 \\
0 & 0 \\
0 & 0 \\
\hdashline & $=$
\end{tabular} & $\overrightarrow{\bar{\Delta}}$ \\
\hline & & $\simeq$ & 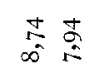 & 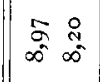 & 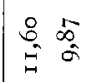 & $\begin{array}{ll}8 \\
8 \\
0\end{array}$ & 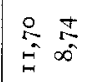 & 点怘 & 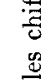 \\
\hline & & $\ddot{z}$ &  &  & $\begin{array}{ll}8 \\
8 \\
0 & 0\end{array}$ & $\mid \begin{array}{ccc}1 & 0 \\
0 & 0 \\
0 & 0 & 0\end{array}$ & 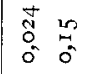 & $\begin{array}{cc}T & 0 \\
0 & 0 \\
0 & 0 \\
0 & 0\end{array}$ & : \\
\hline & & $\frac{\infty}{2}$ & 11 & 吾栗 & $\begin{array}{cc}0 & m \\
0 & 0 \\
0 & 0 \\
i n\end{array}$ & $\begin{array}{ll}\infty & y \\
0 & 0 \\
0 & 0\end{array}$ & 点管 & 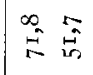 & 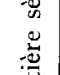 \\
\hline & \multicolumn{2}{|c|}{ 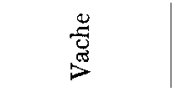 } & $t \infty$ & $1+1$ in & $\exists \Leftrightarrow$ & $\rightarrow$ w & 十曰 & 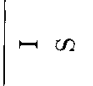 & $\|$ \\
\hline & \multicolumn{2}{|c|}{$\stackrel{\mathscr{\Xi}}{\sharp}$} & 总号 & 吾 & $\frac{9}{0}$ & 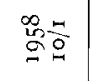 & $\frac{a}{\not D}$ & $\frac{3}{3}$ & \\
\hline
\end{tabular}


endocrine. Le calcul de la signification de l'écart entre le point critique et sa valeur dans le cas d'une hyperbole montre qu'il n'est pas possible de placer ce point sur la courbe théorique.

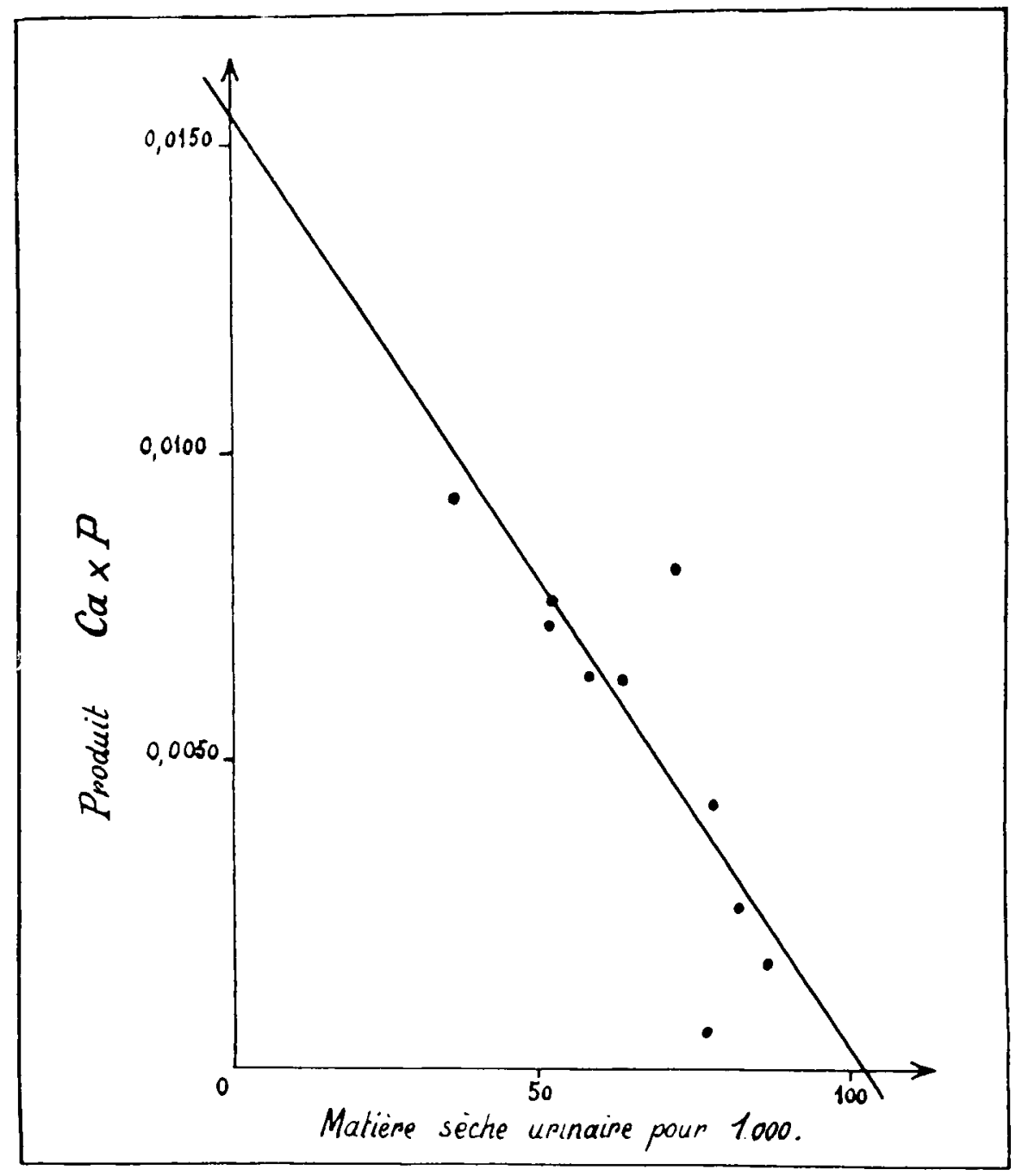

Fug. 3. - Régression : matière sèche urinaire - produit $\mathrm{Ca} \times \mathrm{P}$.

On sait, par ailleurs, qu'il a été démontré par de nombreux travaux que le seuil rénal d'élimination des phosphates est sous la dépendance de l'hormone parathyroïdienne (AlBRIGHT et REIFENSTEIN, I948). on est donc autorisé à penser que la brusque augmentation de la concentration urinaire du phosphore observée lorsque le taux calcique urinaire est inférieur à 0,03 correspond à l'entrée en jeu d'une sécrétion parathyroï- 
dienne accrue. Un taux calcique urinaire égal ou inférieur à 0,03 pourrait donc constituer un des éléments d'évaluation de l'activité parathyroidienne.

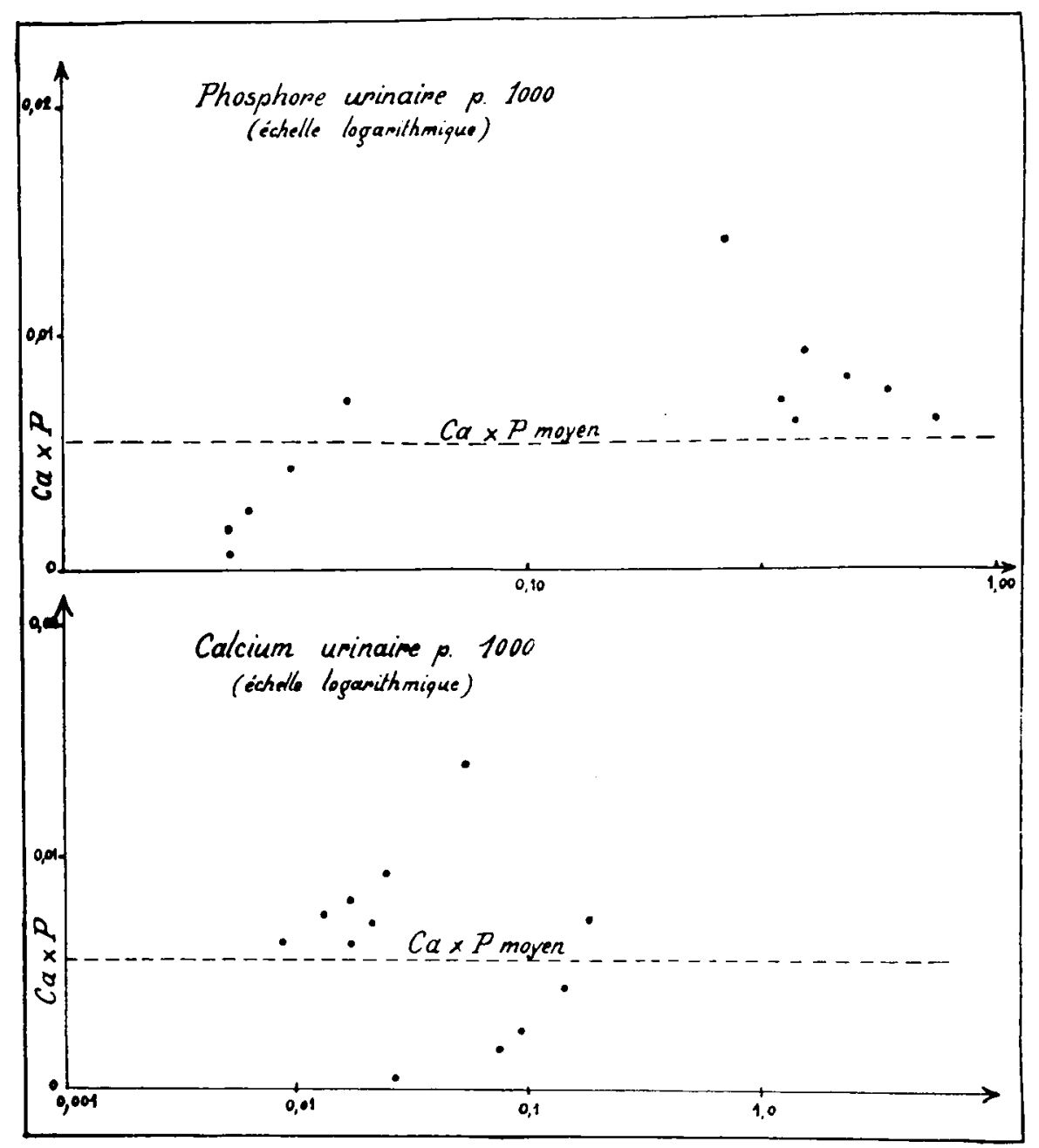

FIg. 4. - Relation entre le produit $\mathrm{Ca} \times \mathrm{P}$ urinaire et le $\mathrm{Ca}$ ou le $\mathrm{P}$ urinaire.

Pour la détermination du produit $\mathrm{Ca} \times \mathrm{P}$ il est indispensable d'avoir un échantillon moyen de l'urine de $24 \mathrm{~h}$.

$2^{\circ}$ Rapports de $\mathrm{Na}$ et $\mathrm{K}$ urinaires.

Si nous considérons l'ensemble des courbes nycthémérales de concentration urinaire, nous observons tantôt un parallélisme de la concentration en sodium et potassium, tantôt une relation inverse, tantôt pas de relation apparente. Ainsi, chez la génisse $S$, à la date du 7 novembre 


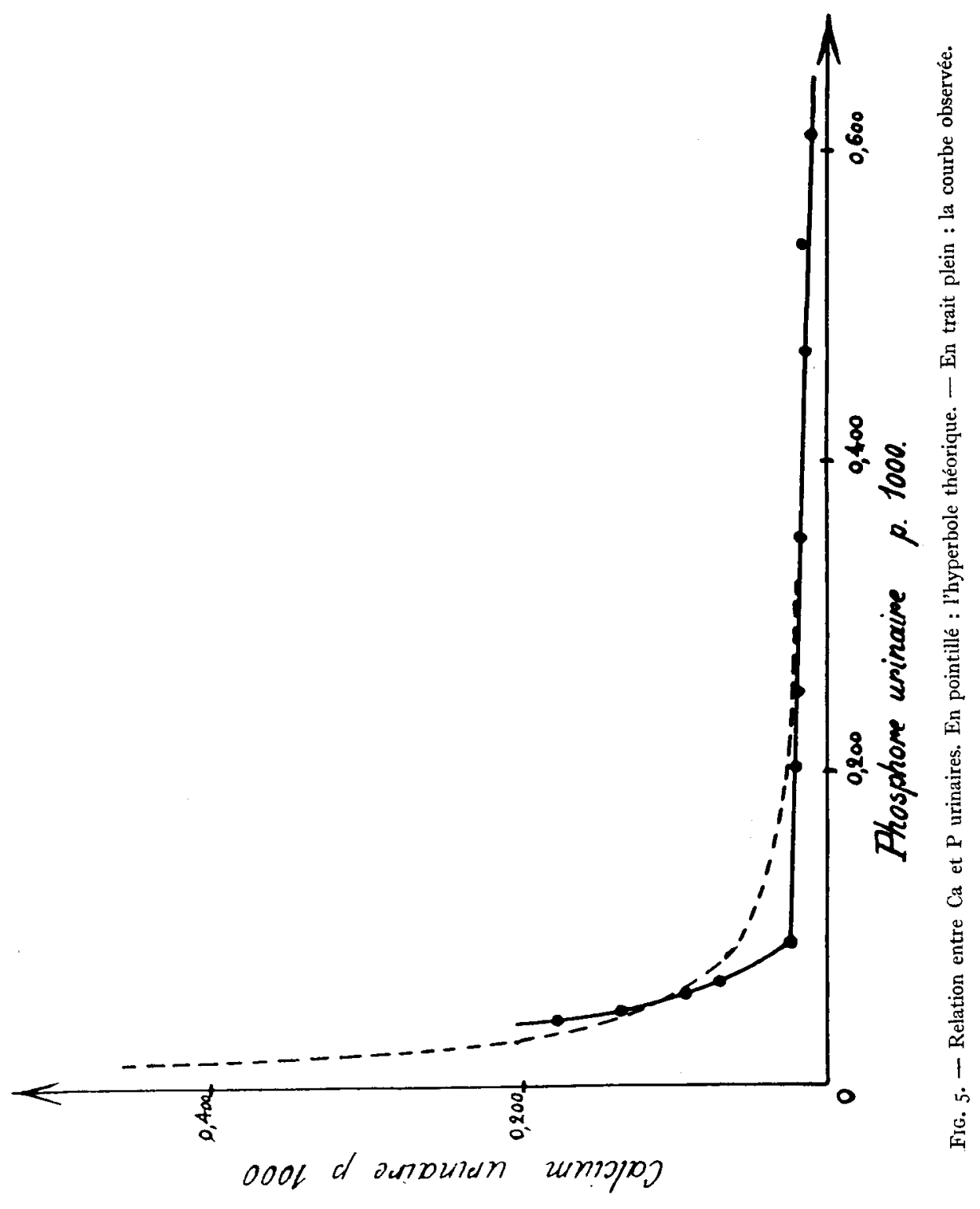


(fig. 6), la corrélation est négative et très suggestive encore que non significative, vu le peu de degrés de liberté. Par contre, les courbes du 6 décembre, pour la génisse III (fig. 6) présentent une corrélation positive significative à I p. Ioo.

L'hypothèse la plus vraisemblable est que, dans ce dernier cas, l'activité minérale de la surrénale n'a que faiblement varié, alors que,

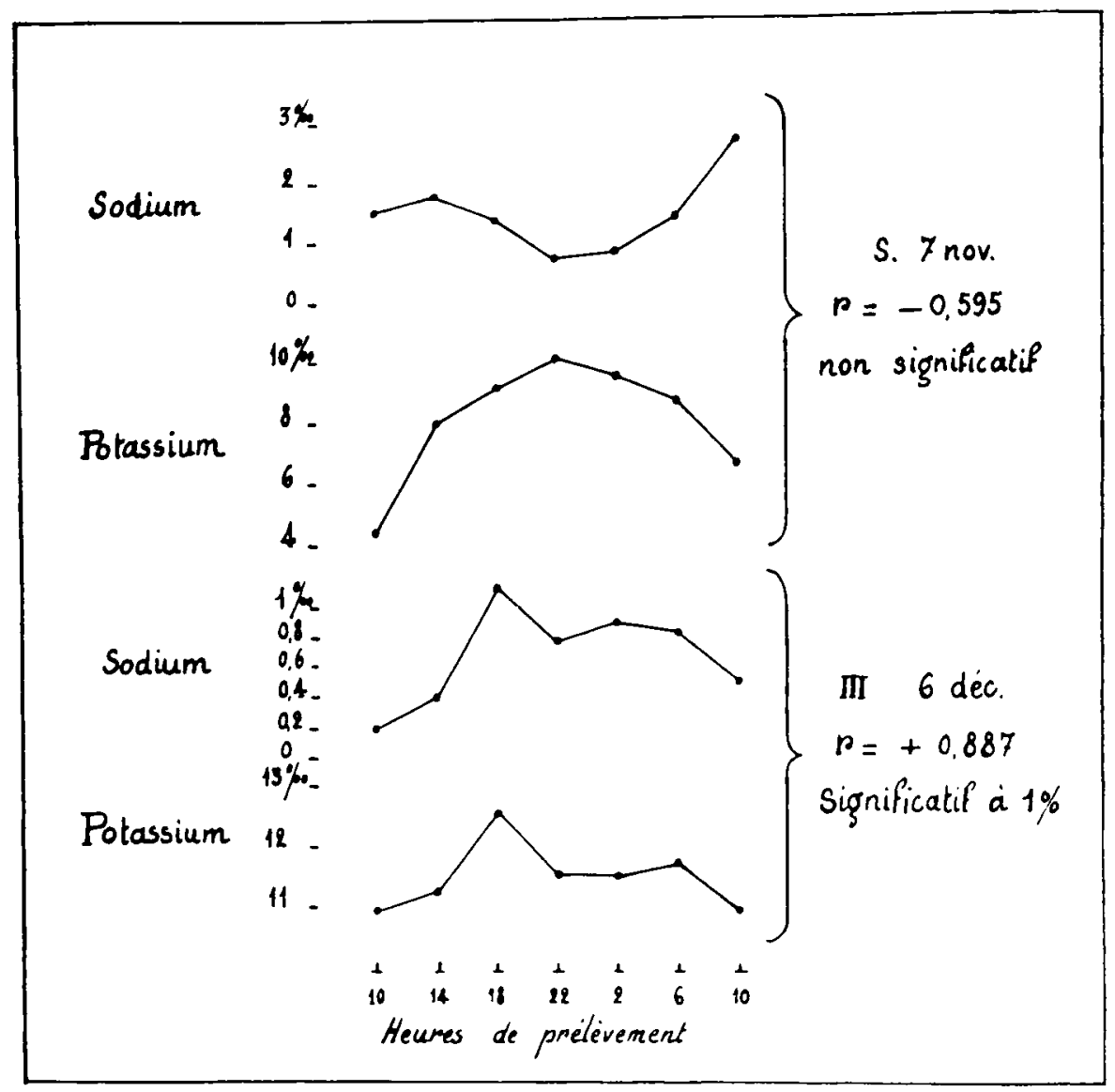

FIG.6. - Relation tantôt positive tantôt négative, entre le sodium et le potassium urinaire au cours d'un même nycthémère.

dans le cas précédent, on assiste à une variation particulièrement nette du taux des minéralocorticoïdes; cette interprétation suppose que l'on considère le rapport $\mathrm{K} / \mathrm{Na}$ comme représentatif de l'activité aldostéronique et de ses variations même au cours d'un nycthémère, ce qui n'est pas démontré et fera l'objet d'une discussion au paragraphe V. 


\section{V. - DISCUSSION DES RESULTATS}

Il existe un certain nombre de travaux portant sur le rythme nycthéméral des bovins ; cependant, nous n'avons pas trouvé, dans la littérature, d'études portant sur le rythme d'élimination ou de concentration de l'urine ou des fèces.

Bien que, selon Johnston et Coll. (I95I), le test de l'éosinopénie après décharge de corticoïdes soit également valable chez les bovins, Schultz (I905), Romano (I935) et Moberg (I955) n'ont pu mettre en évidence une variation nychtémérale du taux d'éosinophiles sanguins. Il est vrai que les mesures de MOBERG ne sont faites qu'entre 5 et I 7 heures, et qu'il note un léger minimum des éosinophiles vers I4 heures, mais non significatif.

L,es tentatives de mesure directe de l'élimination urinaire des corticoides n'ont donné que peu de résultats, par suite de difficultés techniques; Meschaks (I948), Mixner et Coll. (I957), et Estergreen et Coll (I957) dosent les "stérö̈des neutres " urinaires par des techniques dérivées de la réaction de ZIMMERMann et observent une variation nycthémérale, tandis que HoL,Tz (I954) démontrait que la réaction de ZimmermanN est sans valeur chez les bovins, dont l'urine contient une grande quantité d'ionones provenant du métabolisme des caroténoïdes et qui donnent également une coloration intense par ce procédé. D'ailleurs, Fistergreen et Coll. reconnaissent que l'injection d'A. C. T. H. à leurs bovins n'a pas été suivie d'un accroissement urinaire des " stéroïdes neutres ", et ils concluent que les "stéroides urinaires neutres " qu'ils ont dosés ne paraissent pas refléter efficacement les stéroides surrénaux. C'est pourquoi nous estimons que, chez les bovins, les seuls résultats qui puissent être pris en considération sont ceux de Holcombe (I957) ; cet auteur après une étude de l'excrétion urinaire des stéroïdes chez des animaux surrénalectomisés avec ou sans thérapeutique substitutive (cortisone + D. O. C. A.), étude effectuée simultanément avec plusieurs techniques de dosage, a démontré que la technique de SPRECHLER de dosage des " corticoïdes réducteurs " représentait le plus fidèlement l'excrétion des stéroïdes d'origine surrénalienne chez les bovins. Il observe chez le taureau un maximum d'excrétion double, l'un à 8-io heures, l'autre, plus élevé, à I6-I 8 heures; le minimum est situé entre 24 et 7 heures. Ces résultats sont à rapprocher de ceux de VESTERgaARD et LEVERETT, qui trouvent aussi chez l'homme un double sommet d'excrétion.

Nos résultats ne sont pas compatibles avec l'éventualité d'une action déterminante des corticoïdes dosés par HoLCombe sur l'excrétion des ions alcalins, et plus particulièrement du sodium. En effet, les travaux de 
SARTORIUS et Roberts (I949) sur l'action conjointe de l'A. D. H. et de la D. O. C. A., montrent que ces deux hormones sont antagonistes sur l'excrétion de sodium et synergiques sur l'excrétion de potassium, et MOREL (I953) constate que l'A. D. H. "exerce une action inhibitrice directe sur la réabsorption du sodium indépendante de son action antidiurétique, et antagoniste de l'augmentation de réabsorption sodique provoquée par la $\mathrm{D}$. O. C..

Or, la concentration minimum en sodium se situe à 22 heures, heure à laquelle la matière sèche urinaire (donc la sécrétion d'A. D. H. ; Mills, 195I) est élevée, et la sécrétion de corticoïdes faible (HoLcombe); il y a donc deux raisons théoriques pour que le taux de sodium urinaire soit élevé. DoE et Coll. (I956) avaient noté que, si l'excrétion urinaire des I7 cétostéroïdes chez 1'homme est parallèle à celle du potassium,l'excrétion du sodium ne présente par contre aucune corrélation. Ils en concluent que les corticoïdes jouent un rôle dans l'excrétion du potassium, mais non dans l'excrétion du sodium, dissociation qui est en contradiction avec les données physiologiques les mieux établies. Cette excrétion paradoxale du sodium pourrait s'expliquer par l'influence prépondérante sur le métabolisme sodique et potassique d'un corticoïde très actif physiologiquement mais peu important pondéralement : l'aldostérone. L'existence d'un rythme nycthéméral de 1'aldostérone indépendant de celui des autres stéroïdes semble nettement suggérée par l'allure de la courbe de $\log \mathrm{K} / \mathrm{Na}$ urinaire. On sait que Chersin et Coll. (I950) ont établique ce rapport est en corrélation étroite avec le S. R. F. ( $\left.{ }^{(}\right)$excrété. I a validité de cette corrélation peut-elle être étendue au rythme nycthéméral? Cette hypothèse séduisante ne semble pas étayée par les travaux effectués jusqu'à présent. Selon Garrod, Simpson et TAIt (I956), il y a chez l'homme un contraste entre l'importance des variations nycthémérales du rapport $\mathrm{K} / \mathrm{Na}$ et l'absence de variation de l'aldostéronurie. Par ailleurs les travaux de Venning et Coll. (1954-56) étudiant les différences d'élimination urinaire de l'aldostérone entre le jour et la nuit chez l'homme, obtiennent des résultats assez variables et non significatifs statistiquement malgré le nombre de cas étudiés, ce qui est en opposition avec la netteté du rythme des glucocorticoïdes chez les mêmes sujets et démontre l'autonomie de sécrétion de l'aldostérone. Il n'est d'ailleurs nullement certain que 1'aldostéronurie soit le reflet fidèle de l'aldostéronémie, et il n'est pas exclu qu'une augmentation nocturne de cette dernière ne se traduise pas au niveau urinaire, par suite de la diminution générale de l'excrétion des métabolites pendant cette période. En définitive, le rôle directeur de l'hypothalamus, même en ce qui concerne l'aldostérone (RAuschKOLB et FARREL, I956), ne semble pas faire de doute.

(1) Sodium retaining factor $=$ aldostérone. 
On a vu que l'évolution du Ca urinaire (fig. I) coïncide en général avec celle de la matière sèche; le taux calcique urinaire semble donc dépendre essentiellement, au cours du nycthémère, du degré de dilution

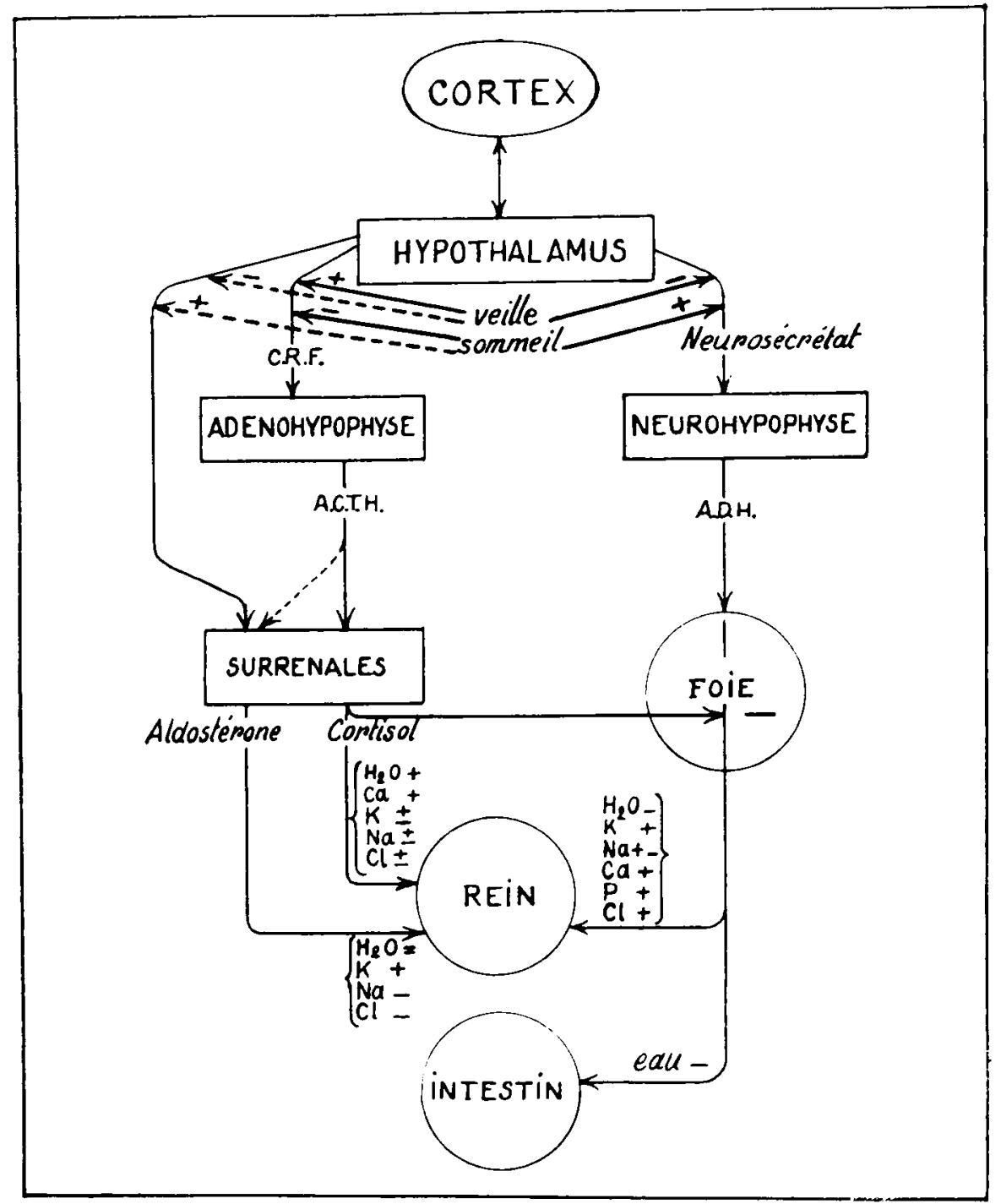

FIG. 7. - Interactions hormonales dans le déterminisme du rythme nycthéméral. Les indications sur l'élimination des minéraux ne concernent que leur concentration urinaire et non les quantités absolues excrétées. (C. R. F. = Corticotrophin Release Factor).

de l'urine, donc de la sécrétion d'A. D. H. On observe toutefois, à 22 h., une certaine diminution du taux calcique, alors que la M.S. est maximum à cette heure, le taux sodique étant par contre minimum ; si l'on admet que le taux sodique (ou que $\log \mathrm{K} / \mathrm{Na}$ ) est une expression valable 
de la sécrétion d'aldostérone, on peut envisager que cette hormone exerce une action inhibitrice sur l'élimination urinaire calcique, la cortisone ayant une action en sens inverse (CLARK et GEOFFroy, I957).

I a figure 7 représente le schéma des interactions hormonales, telles qu'on peut actuellement les envisager, dans le déterminisme du rythme nycthéméral.

En ce qui concerne le rythme fécal de concentration, nous pensons qu'il est déterminé essentiellement par l'évolution de la température extérieure, soit que l'augmentation de la température diminue la résorption des minéraux, soit qu'elle favorise leur excrétion d'origine endogène. On notera que tous les minéraux ne semblent pas être influencés au même titre par les variations de température; alors que les concentrations en $\mathrm{Na}, \mathrm{K}$ et $\mathrm{P}$ évoluent parallèlement à la température, la concentration fécale en Ca varie peu, et ne suit pas le mouvement des autres électrolytes.

La teneur en eau des fécès peut être commandée par un mécanisme endocrinien ; DONNET, Garnier et MAFFre (I95 I a et b, I953, I956) ont mis en évidence chez le chien la possibilité d'un tel mécanisme. Selon ces auteurs, 1'A. D. H. accroît la résorption intestinale de 1'eau, ce qui correspondrait bien à l'augmentation nocturne de la matière sèche fécale et à sa diminution matinale.

I,étude des variations de $\log \mathrm{K} / \mathrm{Na}$ urinaire nous a montré qu'une corrélation hautement significative ( $\mathrm{r}=0,984$ pour $\mathrm{I} 2$ degrés de liberté) lie la valeur de ce rapport prise à $\mathrm{I}_{4}$ heures et la moyenne du nycthémère. Pat ailleurs, Chernik et Coll. (I950) et Luerscher (I956) ont établi qu'une corrélation étroite lie la quantité urinaire de S. R. F. (c'est-à-dire l'aldosterone) et l'inverse de la quantité de sodium excrété, ou le logarithme du rapport $\mathrm{K} / \mathrm{Na}$ urinaire. Il en résulte que le $\log \mathrm{K} / \mathrm{Na}$ d'un échantillon urinaire pris à 14 heures peut représenter valablement (encore qu'approximativement) un indice de l'activité minéralocorticoïde d'un organisme, dans des conditions de nutrition minérale déterminées.

Nous n'avons pas constaté, d'un nycthémère à l'autre, de variation dans l'excrétion fécale de sodium ou de potassium en fonction de l'activité minéralocorticoïde telle qu'elle peut être définie par le $\log \mathrm{K} / \mathrm{Na}$ urinaire.

Nous tenons à remercier MM. Pol,y et Vissac, du Laboratoire de Zootechnie de 1'Institut National Agronomique (I. N. R. A.) pour l'aide qu'ils nous ont apportée dans l'interprétation statistique de ces résultats.

\section{RESUME}

I. - L'évolution de la concentration urinaire et fécale en $\mathrm{Na}$, $\mathrm{K}, \mathrm{Cl}, \mathrm{Ca}, \mathrm{P}$, a été déterminée au cours de six périodes de 24 heures; les 
prélèvements ont été effectués toutesles 4 heures. Quatre génisses ont été utilisées pour ces expériences, deux d'entre elles faisant l'objet de chaque période expérimentale, soit douze observations au total.

2. - La matière sèche urinaire présente un rythme nycthéméral net, avec un minimum à ro heures et un maximum à 22 heures; ce phénomène est probablement sous l'influence de l'hormone antidiurétique. La concentration urinaire en potassium et le $\log \mathrm{K} / \mathrm{Na}$ évoluent de façon sensiblement parallèle à la matière sèche. Par contre, le sodium et le chlore présentent une évolution diphasique nette, avec un minimum à 22 heures et deux maxima, à $\mathrm{I} 4$ heures et 2 heures pour $\mathrm{Na}$, à $\mathrm{I} 8$ heures et 6 heures pour Cl. L'évolution de la concentration urinaire en Calcium au cours du nycthémère est également légèrement biphasique. La courbe du phosphore s'abaisse lentement entre I4 et $I 6$ heures, après une élévation rapide entre 6 heures et 14 heures.

3. - La matière sèche fécale présente un rythme nycthéméral remarquablement régulier, avec un minimum à ro heures et un plateau de I4 à 6 heures. Le sođium, le potassium et le phosphore fécal varient parallèlement à la température extérieure ; le calcium fécal varie peu et de façon apparemment indépendante.

4. - Les coefficients de variation des concentrations urinaires aux différentes heures ont été déterminés afin d'établir s'il existe, au cours du nycthémère, des heures privilégiées, conférant à un prélèvement unique un caractère plus représentatif qu'à d'autres heures. Dans les conditions de l'expérience, les périodes suivantes paraissent les plus favorables :

Matière sèche, $\mathrm{Ca}, \mathrm{Cl}, \mathrm{K}:$ I4 h; $\mathrm{Na}, \mathrm{P}$ : I8 h.

Les cœfficients de corrélation suivants ont été observés entre la valeur isolée à l'heure où le cœfficient de variation est minimum et la valeur moyenne du nycthémère :

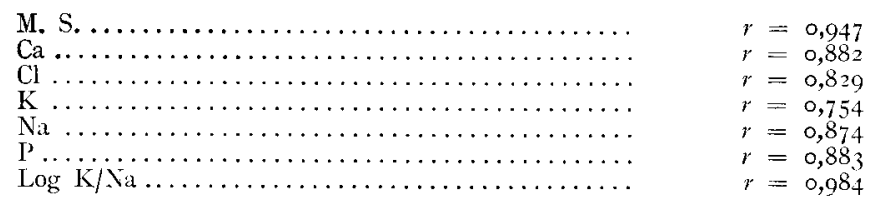

pour Io degrés de liberté (Seuil de signification à I p. Ioo $=0,708$ ).

5. - Une étude analogue effectuée sur les concentrations fécales en $\mathrm{K}, \mathrm{Na}, \mathrm{Ca}, \mathrm{P}$ montre que la dispersion des valeurs est plus faible que pour l'urine. L'heure du prélèvement est sans influence en ce qui concerne la matière sèche, $\mathrm{Ca}$ et $\mathrm{P}$; pour $\mathrm{K}$ et $\mathrm{Na}$, il est préférable d'effectuer les prélèvements vers $\mathrm{I} 8$ heures. 
L,es coefficients de corrélation entre la mesure effectuée à I 8 heures et la moyenne du nycthémère sont :

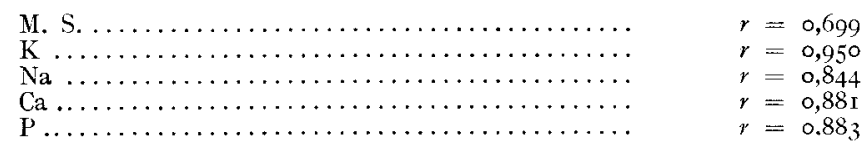

pour Io degrés de liberté.

6. - Le produit des moyennes par nycthémère de $\mathrm{Ca}$ et $\mathrm{P}$ urinaire tend à être constant ; une régression linéaire lie ce produit $\mathrm{Ca} \times \mathrm{P}$ et la matière sèche urinaire ( $\mathrm{Ca} \times \mathrm{P}=-0,000 \mathrm{I}_{52} \mathrm{M}$. S. + o,oI54).

La corrélation entre $\mathrm{Ca}$ et $\mathrm{P}$ urinaires, corrigée en fonction de la matière sèche, peut être exprimée par l'équation :

$$
\mathrm{Ca}=\frac{0,0055}{\mathrm{P}}
$$

La courbe réelle présente toutefois un point d'inflexion pour une valeur de $\mathrm{Ca}$ urinaire de $0,03 \mathrm{p}$. Iooo qui peut correspondre à un seuil de déclenchement de l'activité parathyroïdienne.

7. - La relation qui lie, au cours d'un même nycthémère, le Na et le $\mathrm{K}$ urinaire est tantôt positive, tantôt négative, tantôt inexistante.

8. - Les rôles respectifs de l'hormone antidiurétique, des corticoïdes, de 1'aldostérone, de la parathormone, de la température dans ces différents résultats sont discutés.

Reçu pour publication, le 28 juillet 1958.

\section{RÉFÉRENCES BIBLIOGRAPHIQUES}

(I) Albright (F.) et Reinfenstein (E. C.). - The parathyroid glands and metabolic bone diseases. Ballière, Tyndall et $\mathrm{C}^{\circ}$, édit. London, 1948.

(2) Ames (R. G.) et VAN Dyke (H. B.). - Endocrinol., 50, 350, I952.

(3) D'Arcy Hart (P.) et Verney (E. B.). - Cli. Sci., 1, 367, I934.

(4) Azerad (E.), Ghata (J:) et Reinberg (A.). - Ann. Endocrinol., 18, 484, I957.

(5) Azerad (E.), Lestradet (H.), Reinberg (A.) et Ghata (J.). - Ann. Med., 54, 43I, I953.

(6) Bachman (C.), LEEKLEY (D.) et WinTER (B.). - J. of Clin. Endocrinol., 1, I42, I94I.

(7) Bibile (S. W.). - J. Endocrinol., 9, 357, I953.

(8) Brrnie (J. H.), Eversole (W. J.), Boss (W. R.), Osborn (C. M.) et GaUnt (R.). - Endocrinol., 4\%, I, I950.

(9) Birnie (J. H.), Jenkins (R.), Eversole (W. J.) et Gaunt (R.). Proc. Soc. Exptl Biol. Med., 70, 83, 1949.

(Io) Borst (J. G. G.) et DE VRIES (I. A.). - Lancet, 2, I, 1950.

(II) BrocharT (M.). - Ann. Zootechn., 6, I55, 1957.

(I2) Chernik (S. S.), Chaikoff (I. L.), Masoro (E. J.) et ISAeff (E.). J. Biol. Chem., 186, 527, I950. 
(I3) ClaRK (I.) et GEOFFroy (R.-F.). - Adrenal steroids and Calcium Metabolism in the Rat, Merck Inst. for Therapeutic Research Rahway, N. J., I957.

(I4) Corey (F.), Silvette (H.) et Britton (S. W.). - Am. J. Physiol., 125, 644, I939.

(I5) Coste (F.), Laurent (F.) et Delbarre (F.). - C. R. Soc. Biol., 145, 838, I95I.

(I6) Dicker (S. E.) et Greenbaum (A. L.). - J. Physiol., London, 124, 35, 3P-6P, I954.

(I7) Dicker (S. E.) et Greanbaum (A. L.). - J. Physiol., London, 127 $39 \mathrm{P}, \mathrm{I} 955$.

(i8) Dicker (S. E.) et Greenbaum (A. L.). - J.Physiol., London, 132, I99, I956.

(I9) Dof (R. P.), Flink (E. B.) et Goodseil (M. G.). - J. Clin. Endocrinol. Metab., 16, I96, I956.

(20) Donnet (V.) et Garnier (L.). - Colloque Nat. du C. N. R. S., Marseille, 26-29 janv. I953, p. 205, I953.

(2I) Donne't (V.) et Garnier (L.). - C. R. Acad. Sci., 242, 204 I, 1956.

(22) Donnet (V.), Garnier (L.) et Maffre (S.). - J. de Physiol., 43, 7 I7, I95 I (a).

(23) Donnet (V.), Garnier (L.) et Maffre (S.). - C. R. Soc. Biol., 145, I698, I95I $(b)$.

(24) Elmadjian (F.) et Pincus (G.). - J. Clin. Endocrinol., 6, 287, I946.

(25) Engel (E.), Helmreich (M. L.), Hertoghe (J.) et Borth (R.). - Sem. Hopit., Paris, 31, 70, I955.

(26) Estergreen (V. L. Jr) SONI, (B. K.), Blosser (T.H.) et Erb (R. E.). - Washington Agric. Exp. Sta. Inst. Agric. Sci. State College, Washington. Techn. Bull., 23, II4 pp., I957.

(27) Eversole (W. J.), Birnie (J. H.) et Gaunt (R.). - Endocrinol., 45, 378 , I949.

(28) Forsham (P. H.), Raimondo (V. di), Island (D.), Rinfrei (A. R.) et ORR (R. H.). - Ciba Found. Colloquia on Endocrinol., 8, 279, I955.

(29) Garrod (O.), Simpson (S. A.) et Tait (J. F.). - IVe Congr. Med. Int. Madrid, I956.

(30) Ghata (J.). — Thèse de Doct. en médec., Paris, I954.

(3I) Ghata (J.) et Reinberg (A.). - C. R. Acad. Sci., 239, I680, I954.

(32) GiNSBURG (M.). - J. Endocrinol., 11, I65, I954.

(33) Goldman (R.) et Luchisinger (E. B.). - J. Clin. Endocr. Metab., 16, 28 , 1956 .

(34) Gurnnebauit (M.) et Morel (F.). - C. R. Séances Acad. Sci., 22, 274I, 1957.

(35) HALBERG (F.). - J. Lancet, 73, 20, I953.

(36) Halberg (F.), Visscher (M. B.), Finink (E. B.), Berge (K.) et Bock (F.). - J. Lancet, 71, 3I2, I95I.

(37) Hechter (O.), Frank (E.), Caspi (E.) et Frank (H.). - J. Clin. Endocrinol., 16, $9 \mathbf{I} 6$, 1956.

(38) Heller (H.) et URBAN (F. F.). - J. Physiol., London, 85, 502, I935.

(39) Helier (M.) et ZAIdi (S. M. A.). - Brit. J. Pharmacol. et Chemiother., $12,284,195^{\mathrm{e}}$.

(40) Herbert (P. H.) et de Vries (J. A.). - Endocrinology, 44, 259, I949.

(4I) Holcombe (R. B.). - Acta Endocrinol., Suppl. 34 (accompagne vol. 26), IOO pp., I957.

(42) Holm (L. N.) et Firch (G.). - Am. j. Vet. Res., 16, 332, I955.

(43) Houtz (A. H.). Nature, 174, 3I6, I954.

(44) Johnston (J. E.), Rusoff (L. L.) et d'Arensbourg (G.). - J. Dairy Sci., 34, 497, I95I.

(45) Jores (A.). - Dtsch. Arch. klin. Med., 175, 244, I933.

(46) Kayser (C.). - J. Physiol. (Paris), 41, IA, I949. 
(47) KLEITMAN (N.), - Sleep and wakefulness as alternating phases in the cycle of existence. Univ. of Chicago Press, Chicago, 1939.

(48) Kleitman (N.). - Physiol. Revs., 29, I, 1949.

(49) Laidlaw (J. C.), Jenkins (D.), Reddy (W. J.) et Jakobsox (T.). J. Clin. Invest., 33, 950, I954.

(50) L.eAthes (J. B.). - J. Physiol., 35, I25, I906-07.

(5I) Loiseleur (J.). - Techniques de laboratoire, I vol. in-8 ${ }^{\circ}, 907 \mathrm{pp}$,

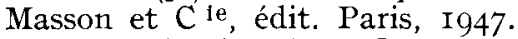

(52) Luetscher (J. A. Jr). - Recent Progr. in Hormone Res., 12, I75, 1956.

(53) Manchester (R. C.). - J. Clin. Invest., 12, 995, I933.

(54) Meschaks (P.). - Skand. Vet. -tidskr.,38, 278, 1948.

(55) Metz (B.) et Mours-Laroche (M. F.). - J. Physiol. (Paris), 46, 464, I954.

(56) Mills (J. N.). - J. Physiol. (London), 113, 528, I95I.

(57) Mixner (J. P.), Saunders (H. L.) et Johnston (J. E.). - J. Dairy Sci., 40, 66, I957.

(58) MobERG (R.). - "The white blood picture in sexually mature female cattle with special reference to sexual conditions". (Thèse Vet. Stockholm), I955.

(59) Morer, (F.). - Colloque du C. N. R. S. "L'équilibre hydrominéral de l'organisme et sa régulation ", p. I63, I953.

(6o) Noble (S.). - Proc. Soc. Exptl. Biol. Med., 95, 679, I957.

(6I) Norn (M.). - Skandinav. Arch. f. Physiol., 55, I84, I929.

(62) Pincus (G.). - J. Clin. Endocrinol., 3, I95, I943.

(63) Pincus (G.), Romanoff (L. P.) et Carlo (J.). - J. Clin. Endocr., 8, 22I, I948.

(64) Rarit (E. P.), Robson (J. S.), Clarke (D.) et Hoagrand (C. L.). J. Clin. Invest., 24, 3I6, I945.

(65) Rauschkolb (E. W.) et FarReli (G. L.). - Endocrinology, 59, 526, 1956.

(66) Romano (J.). - - Beiträge zur Kenntnis des quantitativen und qualitativen Blutbildes (ohne die Thrombozyten). Inaug. Diss. Zagreb (cité par MOBERG).

(67) Rosenbaum (J. D.), Ferguson (B. C.), Iavis (R. K.) et Rossmeisi. (E. C.). - J. Clin. Invest., 31, 507, 1952.

(68) Rud (F.). - Acta psychiat. et neurol., Suppl. 40, pp. I-443. I947.

(69) SartoriUs (O. W.) et Roberts (K.). - Endocrinology, 45, 273, I949.

(70) Schultz (K.). - Untersuchungen uiber das Verhalten der Leukozyten der Wiederkäuer unter normalen Verhältnissen und bei innerlichen Krankheiten, spez. bei Gastritis und Pericarditis. (Inaug. Diss. Bern). Cité par MOBERG.

(7I) Silivette (H.) et Britton (S. W.). - Am. J. Physiol., 104, 399, 1933.

(72) Silvette (H.) et Britton (S. W.). - Am. J. Physiol., 123, 630, I938.

(73) Srmpson (G. E.). - J. Biol. Chem., 59, 107, I924.

(74) Simpsox (G. E.). - J. Biol. Chem., 6\%, 505, 1926.

(75) Simpson (G. E.). - J. Biol. Chem., 84, 393, I929.

(76) Simpson (S. A.) et Tait (J. F.). - Endocrinology, 47, 308, I950.

(77) Sirota (J. H.), Baldowin (D. S.) et Villareal, (H.). - J. Clin. Invest., 29, I87, I950.

(78) Stanbury (S. W.) et Thomson (A. E.). - Clin. Sci., 10, 267, I95I.

(79) Stutinsky (F.). - Rapports de la IVe Réunion des endocrinologistes de langue française, Paris, 27-29 juin I957, p. I93., I957.

(8o) Tyler (F. K.), Migeon (C.), Florentin (A. A.) et Samuels (L. T.). - J. Clin. Endocrinol. Metab., 14, 7, I954.

(8I) Venning (E. H.), Dyrenfurth (I.) et Giroud (C. J. P.). - Fed. Proc., 14, 297, I954.

(82) Venning (E. H.), Dyrenfurth (I.) et Giroud (C. J. P.). - J. Clin. Endocr. Metab., 16, I326, I957.

(83) VestergaARd (P.) et LEVERETT (R.). - Acta Endocrinologica, 25, 45, 1957.

(84) Weish (J. H.). - Quart. Rev. of Biol., 13, I23, I938. 